\title{
The early universe with high-scale supersymmetry
}

\author{
Sibo Zheng ${ }^{\mathrm{a}}$ (1) \\ Department of Physics, Chongqing University, Chongqing 401331, People's Republic of China
}

Received: 15 April 2015 / Accepted: 12 August 2015 / Published online: 25 August 2015

(C) The Author(s) 2015. This article is published with open access at Springerlink.com

\begin{abstract}
A small tensor-to-scalar ratio $r$ may lead to distinctive phenomenology of high-scale supersymmetry. Assuming the same origin of SUSY breaking between the inflation and visible sector, we show model independent features. The simplest hybrid inflation, together with a new linear term for the inflaton field which is induced by a large gravitino mass, is shown to be consistent with all experimental data for $r$ of order $10^{-5}$. For superpartner masses far above the weak scale we find that the reheating temperature after inflation might be below the value required by thermal leptogenesis if the inflaton decays to its products perturbatively, but above it if the decay is non-perturbatively instead. Remarkably, the gravitino overproduction can be evaded in such high-scale supersymmetry because of the kinematically blocking effect.
\end{abstract}

\section{Introduction}

After the discovery [1,2] of the standard model (SM) Higgs boson at the large hadron collider (LHC), low-scale supersymmetry (SUSY) (see, e.g. [3]), which is favored by the naturalness argument (see, e.g. [4]) has been extensively explored. These studies show the difficulties in both the theoretic explanation of the $125-\mathrm{GeV}$ Higgs mass and the experimental fits to the LHC data. The second run of LHC will shed light on the prospect of such natural SUSY models. Based on the above consideration some efforts have been devoted to the study of high-scale SUSY.

Even though high-scale SUSY cannot be detected at the 14-TeV LHC, it can be still studied via their effects on the evolution of the early universe. Measurement on the tensor-to-scalar ratio $r$ via experiments such as WAMP, Planck, and BICEP, which are devoted to measuring the cosmic microwave background (CMB) temperature anisotropy and polarization during inflation, one may probe high-scale

\footnotetext{
a e-mail: sibozheng.zju@gmail.com
}

SUSY with a mass spectrum far above the weak scale. The measured value of $r$ reported by the Planck Collaboration is of order $r<0.11$ at $95 \%$ CL [5-7], from which the energy scale of inflation can be directly inferred. Since the energy scale of inflation is proportional to $r^{1 / 4}$, it is only mildly sensitive to $r$. So the study of high-scale SUSY remains well motivated as long as $r$ is not extremely small.

In this paper, we consider inflationary models with $r$ far below the Planck bound value $r_{c}=0.11$. The motivation is mainly based on two facts. First, the stability of the SM electroweak vacuum requires $H \leq 0.04 h_{\max }$ [8], where $h_{\text {max }}$ refers to the value $h$ at which the Higgs potential is maximal. For the central value of the top quark pole mass $h_{\max } \sim 10^{10} \mathrm{GeV}$, which implies that $H$ should be smaller than the Planck bound value $H_{c} \sim 10^{16} \mathrm{GeV}$ corresponding to $r_{c}$ (see, e.g. [9]). In this sense a small $r<<r_{c}$ is more favored to guarantee the electroweak vacuum stability against quantum fluctuations during the inflationary epoch. Second, for $r<<r_{c}$, it can still generate a SUSY mass spectrum large enough to escape the LHC constraints.

For simplicity we adopt the assumption that the inflation and visible (namely the minimal supersymmetric standard model, MSSM) sector have the same origin of SUSY breaking. This assumption is rational, as it can be realized in model building. Moreover, it allows us to discuss reheating in the early universe after inflation, once the SUSY mass spectrum and the inflaton decay are identified explicitly.

The paper is organized as follows. In Sect. 2 we reanalyze the model independent consequences from the above assumption within the range $r<<r_{c}$. In Sect. 3, we consider hybrid inflation as an example in the course of highscale SUSY breaking. ${ }^{1}$ We will show that a new linear term for the inflaton field with a large coefficient proportional to $m_{3 / 2}$ affects the inflation significantly, and the simplest hybrid inflation is consistent with $r$ of order $10^{-5}$.

\footnotetext{
${ }^{1}$ For earlier attempts to address this issue, see, e.g. [10,11].
} 
In the second part of this paper we discuss the reheating in the early universe after inflation in Sect. 4. In particular, the reheating temperature $T_{\mathrm{R}}$ after inflation is estimated for a superpartner mass spectrum $m_{0}$ above $\mathcal{O}(100) \mathrm{TeV}$. We find that $T_{\mathrm{R}}$ might be below the value $\sim 10^{9} \mathrm{GeV}$ required by thermal leptogenesis if inflaton decays to its products perturbatively, but above it if the decay is non-perturbatively instead. The gravitino overproduction in conventional highscale SUSY can be easily evaded because of the kinematically blocking effect. Finally, we conclude in Sect. 5.

\section{Implications of the value of $r$ to inflation}

In this section we revise the model independent implications (together with Planck and 9-year WAMP data) to single-field inflation for $r<<r_{c}$. These results provide useful information on the model building of inflation, as a reliable inflation model should at least explain observable quantities as what follows.

(1) First of all the scale of energy density during inflation is directly related to $r$ by

$$
V^{1 / 4}=\left(24 \pi^{2} M_{P}^{4} \epsilon A_{s}\right)^{1 / 4}
$$

where $A_{S}$ is the amplitude of the power spectrum of the curvature perturbation and $\epsilon=r / 16$ in the context of single-field inflation. Recall that $A_{S}^{1 / 2}=H^{2}\left(\phi_{*}\right) / 2 \pi \dot{\phi}$, where $\phi_{*}$ is the value of $\phi$ when wavenumber $k_{*}=$ $0.05 \mathrm{Mpc}^{-1}$ crossed outside the horizon. Substituting the value $A_{S}^{1 / 2} \simeq 3.089 \times 10^{-5}$ reported by the Planck Collaboration [6] into Eq. (1) gives rises to

$$
V^{1 / 4} \simeq 2 \times 10^{16} \cdot\left(\frac{r}{0.20}\right)^{1 / 4} \mathrm{GeV}
$$

Equation (2) is valid independent of the explicit form of the inflation potential. If the MSSM and inflation have the same origin of SUSY breaking, as we have assumed in this paper, the high-SUSY-breaking scale $\sqrt{F}$ in particle physics will be of the order of $\simeq V^{1 / 4}$. For example, $\sqrt{F}$ is of order $\sim 10^{16} \mathrm{GeV}$ for $r \sim 0.1$, and slightly reduced to become of order $\sim 10^{15} \mathrm{GeV}$ for $r \sim 10^{-5}$.

(2) In the context of slow roll inflation the spectral index $n_{S}$ (for scalar) and $n_{t}$ (for tensor) are given by

$n_{s}-1 \simeq 2 \eta-6 \epsilon, \quad n_{t} \simeq-2 \epsilon$,

respectively. Here $\epsilon=\frac{M_{P}^{2}}{2}\left(V_{, \phi} / V\right)^{2}$ and $\eta=M_{P}^{2} V_{, \phi \phi} / V$, with the subscript denoting the derivative of $V$ over $\phi$.
The combination of Planck and 9-year WAMP data measures the value of $n_{s}$ with high precision [7],

$n_{s}=0.9603 \pm 0.0073$.

For $r<<r_{c}$ one finds that $\eta \simeq-0.02$. This tight bound is crucial to constrain the inflation model.

(3) The gravitino mass $m_{3 / 2}$ can be determined. The constant superpotential $W_{0}=m_{3 / 2} M_{P}^{2}$, which is required to cancel a positive $F^{2}$ term in the potential so as to explain the smallness of the cosmological constant, gives rise to

$m_{3 / 2}=\frac{F}{\sqrt{3} M_{P}}$.

(4) Finally the number of e-folds that $k_{*}$ undergoes during inflation is given by

$N \simeq \int_{\phi_{\text {end }}}^{\phi_{\text {in }}} \frac{\mathrm{d} \phi}{M_{P}^{2}} \frac{V(\phi)}{V,(\phi)} \simeq \int_{x_{\text {end }}}^{x_{\text {in }}} \frac{\mathrm{d} x}{\sqrt{2 \epsilon(x)}}$,

where $x=\phi / M_{P}$. The subscripts "in" and "end" correspond to the initial and end value of $x$ during inflation, respectively. For realistic inflation models, $N$ is bounded as $50 \leq N \leq 60$. If $\epsilon$ does not change significantly during inflation, Eq. (6) can be expressed as $\Delta \phi / M_{P} \simeq \sqrt{2 \epsilon} N \simeq \sqrt{\frac{r}{8}} N$. This is known as the Lyth bound [12], which shows the need of small field inflation for $r<<r_{c}$.

\section{The simplest hybrid inflation}

The section is devoted to the study of inflation building in the course of high-scale SUSY. We take the simplest hybrid inflation as an explicit illustration. We will show that a new linear term due to the assumption adopted in this paper significantly affects the choice of the initial condition. Also this assumption introduces new constraints on the parameters in the model, which make the simplest hybrid inflation only possible with $r$ of order $10^{-5}$.

\subsection{Scalar potential}

The scalar potential in hybrid inflation is constructed from superpotential $W$,

$W=\kappa \Phi\left(\bar{\Psi} \Psi-M^{2}\right)$,

and the Kahler potential $K$,

$K=|\Phi|^{2}+|\Psi|^{2}+|\bar{\Psi}|^{2}+k_{1} \frac{|\Phi|^{4}}{M_{P}^{2}}$. 
Here $\Phi$ denotes the inflaton superfield, with its lowest component inflaton field $\phi . \Phi$ is a singlet of the standard model gauge groups $G=S U(3)_{c} \times S U(2)_{L} \times U(1)_{Y} . \Psi$ and $\bar{\Psi}$ denote waterfall superfields, which are in the bi-fundamental representation of $G^{2}$ The Kahler potential in Eq. (8) takes into account the non-canonical term, with $k_{1}$ a real coefficient. The non-canonical $k_{1}$ term provides the inflaton mass term. $M_{P}=2.4 \times 10^{18} \mathrm{GeV}$ is the reduced Planck mass, while $M$ is assumed to be far below $M_{P}$.

Substituting Eqs. (7) and (8) into the SUGRA potential,

$V=e^{K / M_{P}^{2}}\left[K^{i \bar{j}} D_{i} W D_{j} W-3 \frac{|W|^{2}}{M_{P}^{2}}\right]$,

one obtains the scalar potential of hybrid inflation. Here $K^{i \bar{j}}=K_{i \bar{j}}^{-1}$ is the Kahler metric and $D_{i} W=\partial_{i} W+$ $K_{i} W / M_{P}^{2}$. It is well known that the history of inflation can be naturally divided into two periods. In the first one inflation usually starts from an initial value of order $M_{P}$ toward $\phi_{c}$, which is a critical value separating the two periods. The vacuum in the first period corresponds to $\Psi=\bar{\Psi}=0$, from which the energy density reads, from Eq. (9) [13],

$$
\begin{aligned}
V= & \frac{1}{2} m_{\phi}^{2} \phi^{2}+\kappa^{2} M^{4}\left[1+\gamma \frac{\phi^{4}}{8 M_{P}^{4}}+\frac{\kappa^{2}}{16 \pi^{2}} \ln \frac{\kappa^{2} \phi^{2}}{2 \Lambda^{2}}\right] \\
& +2 \sqrt{2} \kappa M^{2} m_{3 / 2} \phi \cos \theta
\end{aligned}
$$

where we have defined $\Phi=\phi e^{i \theta} / \sqrt{2}$. Here $\gamma=1-7 k_{1} / 2+$ $2 k_{1}^{2}$ and the inflaton mass $m_{\phi}$ is given, for negative $k_{1}$, by

$m_{\phi}=\sqrt{-k_{1}} \kappa M^{2} / M_{P}$.

The log-term in Eq. (10) represents the contribution due to mass splitting in waterfall fields [14], with $\Lambda$ the cut-off scale. The linear term with coefficient proportional to $m_{3 / 2}$ arises from a constant superpotential $W_{0}=m_{3 / 2} M_{P}^{2}$ added to $W$, which is needed to cancel a positive contribution to the energy density due to SUSY breaking, and explain the smallness of the cosmological constant.

When $\phi$ approaches $\phi_{c}=\sqrt{2} M, \phi$ becomes massless as shown from its mass squared, $m_{\Psi}^{2}=-4 \kappa^{2} M^{2}+2 \kappa^{2} \phi^{2}$. After the time when $\phi$ is below $\phi_{c}, \Psi$ starts to roll toward its global minimum value $\Psi=M$ from $\Psi=0$, which is known as the second period of inflation. The evaluation of the field $\phi$ (including the angular component $\theta$ ) and $\Psi$ during each period is determined by their equations of motion,

$$
\begin{aligned}
3 H \dot{\phi} & \simeq m_{\phi}^{2} \phi+2 \sqrt{2} \kappa M^{2} m_{3 / 2} \cos \theta, \\
3 H \dot{\theta} & \simeq 2 \sqrt{2} \kappa M^{2} m_{3 / 2} \frac{\sin \theta}{\phi}, \\
3 H \dot{\Psi} & \simeq-2 \kappa^{2}\left(2 M^{2}-\phi^{2}\right) \Psi,
\end{aligned}
$$

\footnotetext{
2 Alternatively, $G$ can be extended to include a local $U(1)_{B-L}$ symmetry so as to explain leptogenesis.
}

where $H$, the Hubble constant, is subject to the Friedmann constraint

$H^{2} \simeq 8 \pi V / 3 M_{P}^{2}$

The time for each period is controlled by the magnitude of $m_{\phi}$ or $\left|m_{\Psi}\right|$ relative to the Hubble constant $H$. As pointed out in [15], the second period is very short compared with the first one for wide ranges of parameter choices. Substituting Eq. (13) into the last equation in Eq. (12), we obtain the constraint for such a property,

$10^{-4}<\kappa<\mathcal{O}(1)$

In the next subsection, we will discuss in more detail the initial conditions on the inflaton field and the field value of $\phi$ when inflation ends.

\subsection{Initial conditions}

The inflation usually begins at some field value $\phi_{\text {in }}$ near the Planck scale. The choice of $\phi_{\text {in }}$ is subtle when the inflaton potential has either a few local minimums at $\phi_{\min } \mathrm{s}$, or local maximum at $\phi_{\max } \mathrm{s}$. If one adopts a value for $\phi_{\text {in }}$ bigger than $\phi_{\text {min }}$, the inflaton is probably trapped at these local minimums of the inflaton potential along the trajectory, which leads to inflation with an insufficient e-fold number $N \sim 50-60$. In order to avoid this, one should choose $\phi_{\text {in }}<\min \left\{\phi_{\min }\right\}$. On the other hand, one wants that inflation proceeds with exactly decreasing $\phi$. This is only allowed if $\phi_{\text {in }}$ is less than $\min \left\{\phi_{\max }\right\}$. In other words, we should impose the initial condition

$\phi_{\text {in }}<\min \left\{\phi_{\max }, \phi_{\min }\right\}$.

In Fig. 1 we show how extremes in $V$ depend on $\cos \theta$ and $\kappa$ by evaluating $\sqrt{2 \epsilon}=V_{, \phi} / V$. The sign of $V_{, \phi} / V$ changes when $x \sim 0.10$ for $\cos \theta=-0.002$ and $x \sim 0.15$ for $\cos \theta=$ -0.003 . This implies that $\cos \theta \simeq 0$ for realistic inflation. Otherwise, $\phi_{\text {in }}<<M_{P}$, which is too small to provide a high enough e-fold number $N$. This observation has been noted in [13] for $m_{3 / 2}$ of the order of the electroweak scale and further verified for a larger value of $m_{3 / 2} \sim 10^{13} \mathrm{GeV}$. With the initial value $\theta \simeq \pi / 2$, the initial value of $\phi_{\text {in }}$ can be chosen in a wide range, as shown in Fig. 1. The evaluation of $\phi$ from $\phi_{\text {in }}$ is the same as in the original hybrid model because of the absence of a linear term in the first equation in Eq. (12). In this sense, inflation mainly ends at the field value $\phi_{\text {end }}=\sqrt{2} M$.

The e-fold number $N$ produced during inflation and $n_{S}$ can both be estimated in terms of the slow roll parameters $\epsilon$ and $\eta$ in the model, which are given by, respectively, 


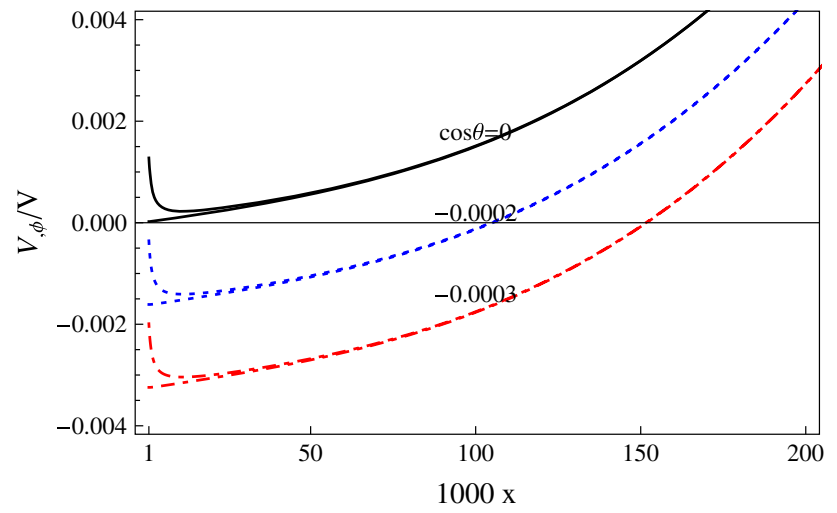

Fig. 1 Initial condition on $x \equiv \phi / M_{P}$ as a function of $\cos \theta$ for $\kappa=$ $0.01,0.001$

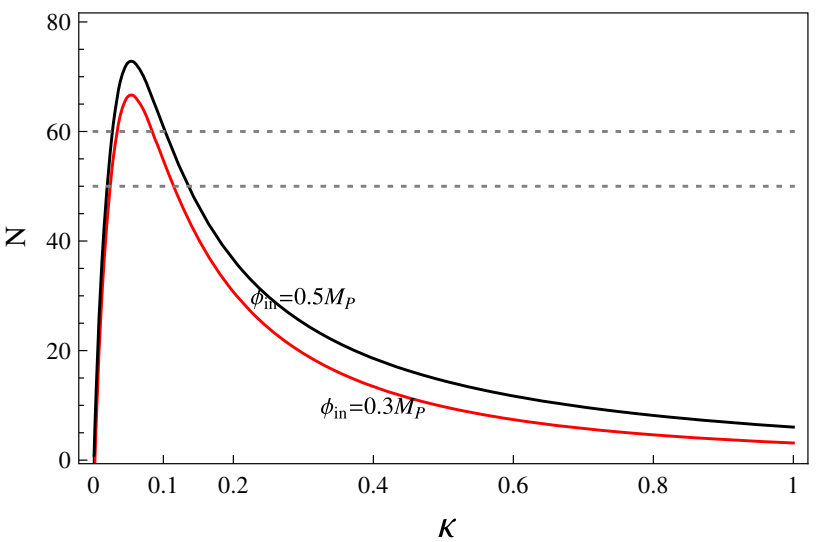

Fig. $2 N$ as a function of $\kappa$ for the initial value $x_{\text {in }}=0.3(\mathrm{red}), 0.5$ (black), respectively. Dotted lines represent the uncertainty of the experimental value. The slow roll condition $|\eta|<1$ leads to the bound $\phi_{\text {in }} \leq M_{P}$

$\eta(x) \equiv M_{P}^{2} \frac{V_{, \phi \phi}}{V} \simeq-k_{1}+\frac{3}{2} x^{2}-\frac{\kappa^{2}}{8 \pi^{2}} \frac{1}{x^{2}}$,

$\epsilon(x) \equiv \frac{M_{P}^{2}}{2}\left(\frac{V_{, \phi}}{V}\right)^{2} \simeq \frac{1}{2}\left(\sqrt{\frac{8}{3}} \cos \theta-k_{1} x+\frac{1}{2} x^{3}+\frac{\kappa^{2}}{8 \pi^{2}} \frac{1}{x}\right)^{2}$.

With $k_{1} \simeq-0.01$ and $\cos \theta \simeq 0, \eta(x)$ and $\epsilon(x)$ mainly depend on the parameter $\kappa$.

Figure 2 shows the bound on $\kappa$ for the two choices of $x_{\text {in }}=$ 0.3 (red curve) and 0.5 (black curve), respectively. Note that $x$ is constrained by the slow roll condition $|\eta|<1$. Given the range shown in Eq. (14) for $\kappa, x$ should be below unity, which implies that large field inflation is excluded under our assumption. Figure 2 shows that $\kappa \sim 0.1$ for $N \sim 50-60$.

In Fig. 3 we show how $n_{s}$ changes for two typical choices of $\kappa$ subtracted from Fig. 2. It clearly indicates that for the observed value of $n_{s}, r$ is of the order $\sim 10^{-5}$. The simplest hybrid inflation can provide a large e-folds number $N \sim 50$ 60 and small $r \sim 10^{-5}$. Nevertheless, large $N$ and large

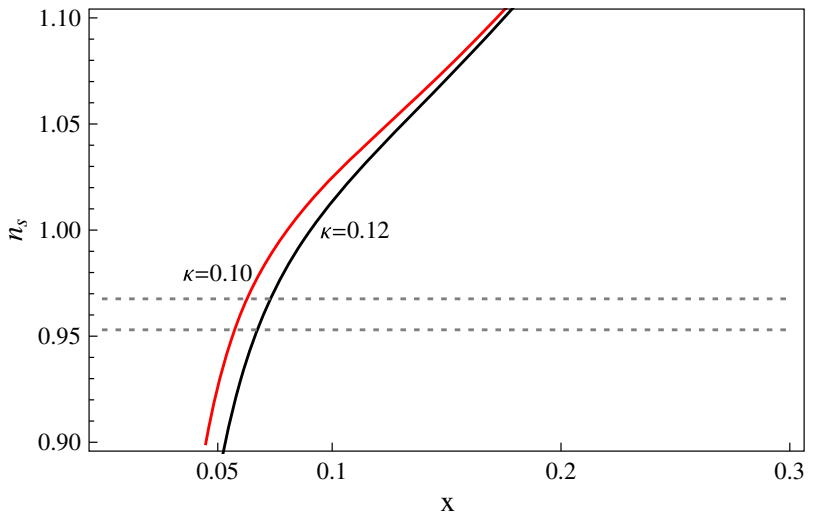

Fig. $3 n_{s}$ as a function of $\kappa$ for the initial value $x_{\text {in }}=0.3($ red $), 0.5$ (black), respectively. Note that $x_{\text {end }} \geq \phi_{c} / M_{P}$. Dotted lines represent the uncertainty of the experimental value

$r>>10^{-5}$ cannot be induced at the same time. In the next section, we focus on reheating after (or during) inflation.

\section{Reheating in high-scale SUSY}

When inflation ends the conversion of energy to the MSSM matter from the inflaton begins immediately. The efficiency of the energy transfer depends on how inflaton is coupled to the MSSM matters, the magnitude of their couplings, and the SUSY mass spectrum. In general, the ways of energy transfer include the perturbative and non-perturbative decay of the inflaton. ${ }^{3}$ The latter way is known as preheating [18-20]. The conditions between these two ways of energy transfer are rather different. In the latter case, parameter resonance requires a quartic interaction term $\sim g^{2} \phi^{2} \chi^{2}$ with large magnitude of $g$. This only happens if one allows a renormalizable superpotential term of mass dimension 4 [21],

$R_{\Phi}=+1: \Phi \mathbf{H}_{u} \mathbf{H}_{d} ; \quad R_{\Phi}=-1: \Phi \mathbf{H}_{u} \mathbf{L}$,

where $\Phi$ is the inflaton superfield, and the $\mathbf{H}_{u, d}$ are Higgs doublet superfields. $R_{\Phi}$ denotes the $R$-parity of the inflaton, which is useful to keep the dark matter stable. In contrast, in the SM the inflaton couples to SM chiral fermions and gauge bosons in terms of non-renormalizable interactions of mass dimension 5. The quartic term above does not exist in the $\mathrm{SM}$, and therefore the way of energy transfer in the SM is perturbative decay. In what follows, we consider these two ways separately.

\subsection{Perturbative decay}

As briefly mentioned above, perturbative decay happens either when there is no renormalizable interaction in Eq. (17)

\footnotetext{
${ }^{3}$ For reviews, see, e.g., $[16,17]$.
} 
or the quartic coupling constant is tiny. Instead, the inflaton only decays to SM matter via five-dimensional operators such as

$$
\left\{\frac{\phi}{M} F_{\mu \nu} F^{\mu \nu}, \frac{\phi}{M} \phi\left(H \bar{q}_{L}\right) q_{R}, \cdots\right\} \text {. }
$$

Here $F_{\mu \nu}$ s refer to the strengths of the SM gauge fields, $q$ s refers to the SM fermions and $M$ represents the mass scale appearing in the five-dimensional operators. The plasma will be MSSM-like if the reheating temperature is larger than the typical scale of the superpartner mass, $m_{0}$. Otherwise, the plasma is actually SM-like.

Now we calculate the reheating temperature. We organize the width of the decay of the inflaton to SM particles as

$\Gamma_{d} \equiv \frac{\lambda}{16 \pi^{2}}\left(\frac{m_{\phi}}{M}\right)^{2} m_{\phi}$.

We simply take $M=M_{P}$ but leave $\lambda$ as a free parameter. The thermal equilibrium of the relativistic plasma is dominated by $\Gamma$, the rate for SM inelastic scatterings of $2 \rightarrow 3$ processes [22,23]. $\Gamma$ is related to $\Gamma_{d}$ by

$\Gamma \sim \alpha^{3}\left(\frac{M_{P}}{m_{\phi}}\right) \Gamma_{d}$,

where $\alpha \sim 1 / 30$ is the SM fine structure constant. Therefore the reheating temperature $T_{R}$ for the perturbative decay is not equal to the conventional one, $T_{r t h}$, defined as $T_{r t h} \simeq$ $0.3 \times\left(\frac{100}{g_{*}}\right)^{1 / 4} \sqrt{\Gamma_{d} M_{P}} ; g_{*}$ denotes the number of relativistic number. Instead, in terms of Eq. (20) we have

$$
\begin{aligned}
T_{R} \simeq & \alpha^{3 / 2} \cdot\left(\frac{2 \pi N_{c}}{0.09} \frac{M_{P}}{m_{\phi}}\right)^{1 / 2} \cdot\left(\frac{g_{*}^{\text {susy }}}{g_{*}^{\text {sm }}}\right)^{1 / 4} \\
& \cdot T_{r t h} \simeq 0.01 \cdot\left(\frac{g_{*}^{\text {susy }}}{g_{*}^{\text {sm }}}\right)^{1 / 4} \cdot\left(\frac{\lambda^{1 / 2}}{4 \pi}\right) \cdot m_{\phi},
\end{aligned}
$$

where $N_{c}$ denotes the quantum numbers of the SM gauge groups. Note that Eq. (21) is valid for $m_{0}<T_{R}$, which implies that the reheating temperature can serve as the upper bound on $m_{0}$.

In Fig. 4 we show the reheating temperature $T_{\mathrm{R}}$ as a function of inflaton mass. Note that $\lambda$ captures the magnitude of coupling between inflaton and "mediate" field, which also couples to the SM matter and gauge fields. Here a few comments are in order. (1) For $\lambda<10^{-3}, T_{\mathrm{R}}$ is below the lower bound $\sim 1 \times 10^{9} \mathrm{GeV}$ required by thermal leptogenesis in the whole range of $m_{\phi}$. (2) Since $T_{\mathrm{R}}$ is the upper bound on the superpartner mass spectrum $m_{0}$, one finds that $m_{0}$ is upper bounded as $1 \mathrm{TeV}<<m_{0}<10^{7} \mathrm{TeV}$ for the case of perturbative decay. (3) As $T_{\mathrm{R}}$ is far below the gravitino mass of Eq. (5), there is no overproduction problem of the gravitino in high-scale SUSY. Superheavy gravitino mass of order $\sim 10^{14} \mathrm{GeV}$ kinetically blocks its production in the thermal bath.

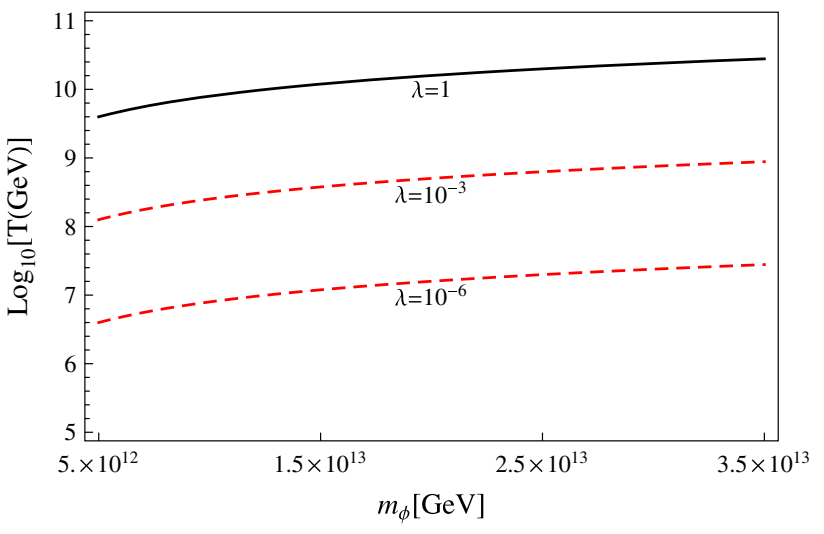

Fig. 4 Reheating temperature (also the upper bound on $m_{0}$ ) as a function of the inflaton mass. The inflaton mass range is inferred as $\sim F / M_{P}$ up to a coefficient

\subsection{Non-perturbative decay}

If it admits a renormalizable superpotential Eq. (17), there exists a quartic interaction between the inflaton and its decay products $\chi_{i}$, and non-perturbative decay can happen in a wide range of the parameter space for the potential of type ${ }^{4}$

$V(\phi, \chi)=\frac{1}{2} m_{\phi}^{2} \phi^{2}+g^{2} \phi^{2} \chi_{i}^{2}+m_{\chi_{i}}^{2} \chi_{i}^{2}$,

where the inflaton mass term is included and $m_{\chi_{i}}$ is the mass for $\chi_{i}$. We would like to mention that $m_{\chi_{i}}$ includes a soft SUSY-breaking contribution of order $\sim m_{0}$ and a dynamical mass $\sim \lambda^{1 / 2}\langle\varphi\rangle$ induced by VEV of the flat direction $\varphi$ $[24,25]$ through the quartic interaction [27],

$V(\chi, \varphi)=\lambda \chi_{i}^{2} \varphi^{2}$,

where $\lambda$ is the quartic coupling constant. The magnitude of $\langle\varphi\rangle$ is determined by the self-interaction potential for the flat direction $V(\varphi)$.

Now we consider the potential for the flat direction. $V(\varphi)$ includes a soft breaking mass, a Hubble parameter induced term, and high dimensional operators,

$V(\varphi) \simeq\left(m_{0}^{2}+c_{H} H^{2}\right) \varphi^{2}+c_{6} \frac{\varphi^{6}}{M^{4}}+\cdots$,

where $c_{H}$ is real coefficient. Since $m_{0}$ is not far below the Hubble constant $H \sim m_{\phi}$ at the beginning of inflation, the VEV $\langle\varphi\rangle$ depends on the sign of $c_{H}$, which can be either positive or negative [24-26]. In particular, $\langle\varphi\rangle=0$ for the case of either positive $c_{H}$ or negative $c_{H}$ but with $\left|c_{H}\right|<<$ 1. It implies that SM gauge symmetry is unbroken during

\footnotetext{
${ }^{4}$ Assuming the inflaton and MSSM matter have the same origin of SUSY breaking, the inflaton mass is dynamically induced by SUSY breaking. In this sense, the mass term $m_{\phi}^{2} \phi^{2}$ is a soft SUSY-breaking term other than the one arising from the SUSY tree-level mass superpotential $\sim m \Phi \Phi$. Consequently, there is no cubic interaction $\phi \chi^{2}$ compared with earlier discussions in [21,27].
} 


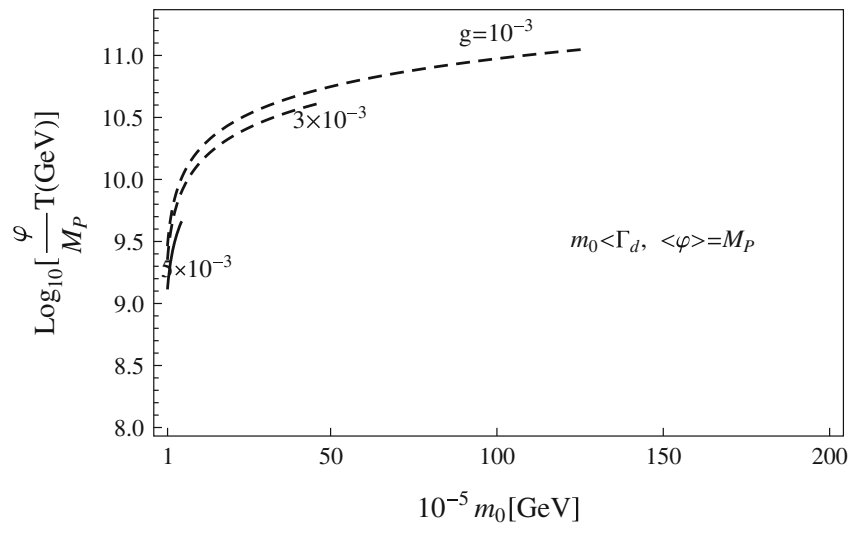

Fig. 5 Reheating temperature as a function of $m_{0}$ for $m_{\phi}=10^{13} \mathrm{GeV}$ and $\langle\varphi\rangle \neq 0$. (Left $m_{0}<\Gamma_{d}$ with $\langle\varphi\rangle=M_{P}$, Right $m_{0}>\Gamma_{d}$ with $\lambda=10^{-3}$ and $\left.\langle\varphi\rangle=0.01 M_{P}\right)$. In the right panel, $\langle\varphi\rangle \geq 0.1 M_{P}$ is

the whole history of the early universe. On the other hand, $\langle\varphi\rangle \neq 0$ for negative $c_{H}$ but with $\left|c_{H}\right|>1$. It implies that SM gauge symmetry is broken in the early universe, with a gauge boson mass of order $\langle\varphi\rangle$, and then is restored after the epoch of reheating.

The potentials we define in Eq. (22) to Eq. (23) are rather general, which can be applied to both cases in Eq. (17). To discuss the condition for parameter resonance, one starts with the modified Klein-Gordon equation for the Fourier modes $\chi$. Whether the WKB approximation is viable for the study can be analyzed in terms of a quantity $R$ defined as [16]

$R \equiv \frac{\dot{\omega}_{k}}{\omega_{k}^{2}}, \quad \omega^{2}=k^{2} / a^{2}+g^{2}\langle\phi(t)\rangle^{2}$,

where a dot refers the to derivative over time and $\omega$ is the frequency, with $a$ the expansion factor and $k$ the momentum. If $|R|<<1$, the WKB approximation is valid, the produced particle number of $\chi$ does not grow in this case. If $|R|>1$ instead, the WKB approximation is not valid, which leads to significant production of $\chi$. In the long wavelength limit, this constraint is given by ${ }^{5}$

$m_{\chi_{i}}^{2} \simeq m_{0}^{2}+\lambda\langle\varphi\rangle^{2}<g^{2}\langle\phi(t)\rangle^{2}$,

where we have used Eqs. (22) and (23). Moreover, in order to keep the parameter resonance from not being spoiled by expansion, an additional constraint must be imposed,

$q \equiv g^{2} \bar{\phi}^{2}(t) / 4 m_{\phi}^{2}>>1$,

where $\bar{\phi}(t) \sim M_{P}$ refers to the amplitude of the inflaton oscillations. For more details, we refer to reader to [16] and references therein.

Figure 5 shows the reheating temperature for the case in which $\langle\varphi\rangle \neq 0$. In this case, SM gauge bosons are massive

\footnotetext{
5 There is a coefficient of order 1 in front of $m_{0}$ for either $R_{\Phi}=1$ or $R_{\Phi}=-1$. Here we simply take it equal to unity.
}

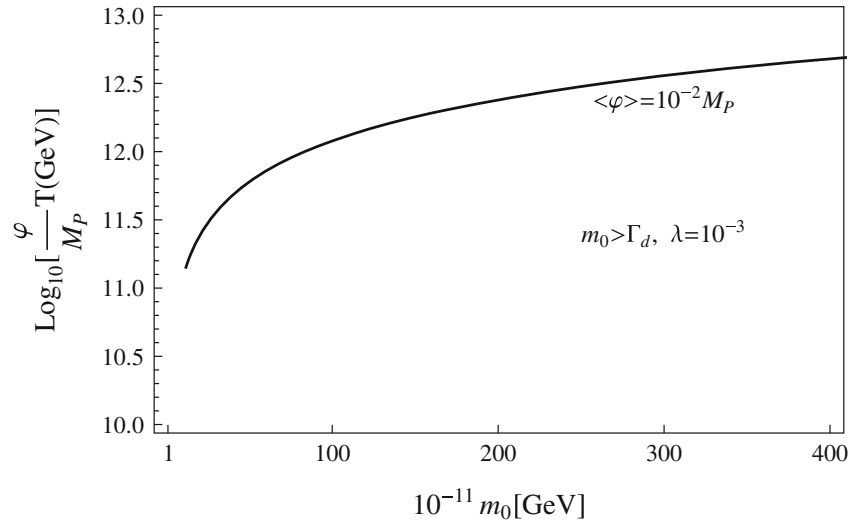

excluded by the condition of Eq. (26) for $\lambda \geq 10^{-3}$. The bounds on $g$ and $m_{0}$ are explained in the text

and the rate for the thermal equilibrium $\Gamma$ [27] depends on its magnitude relative to $m_{0}$. For $m_{0}<\Gamma_{d}, T_{\mathrm{R}}$ depends on both $g$ and $\langle\varphi\rangle$, whereas it mainly depends on $\langle\varphi\rangle$ for $m_{0}>\Gamma_{d}$. In this figure, we take $m_{\phi}=10^{13} \mathrm{GeV}$ and $\bar{\phi}=M_{P}$. The bounds on $g$ are due to a few considerations. The first one is that the condition of Eq. (27) from parameter resonance requires $g>>10^{-5}$. The second one is that the overproduction problem [28] of the gravitino in high-scale SUSY can be kinematically blocked if $g<10^{-2}$ such that the nonperturbatively induced mass during parameter resonance is below $m_{3 / 2} .{ }^{6}$ The bound on $\lambda\langle\varphi\rangle$ arises from the condition of Eq. (26), which shows $\lambda\langle\varphi\rangle^{2}<g^{2} M_{P}^{2}$.

The left panel in Fig. 5 shows that in the range $10^{5} \mathrm{GeV}$ $<m_{0}<1.5 \times 10^{7} \mathrm{GeV}$ the reheating temperature $T_{R} \geq 10^{9}$ $\mathrm{GeV}$ in the allowed range of $g$ for $\langle\varphi\rangle=M_{P}$. We modify $\langle\varphi\rangle<M_{P}$,

$T_{R} \rightarrow\left(\frac{\langle\varphi\rangle}{M_{P}}\right)^{-1} T_{R}$

which is always above the value required by thermal leptogenesis. The right panel in Fig. 5 shows that in the range $10^{11}$ $\mathrm{GeV} \leq m_{0}<4 \times 10^{13} \mathrm{GeV}$ the reheating temperature $10^{11}$ $\mathrm{GeV} \leq T_{R} \leq 10^{13} \mathrm{GeV}$ if $\langle\varphi\rangle=10^{-2} M_{P}$, and it changes similarly to Eq. (28) on modifying $\langle\varphi\rangle$. This implies that $T_{\mathrm{R}}$ is also always above the value required by thermal leptogenesis. Note that $\langle\varphi\rangle \geq 10^{-1} M_{P}$ is excluded by the condition of Eq. (26) from parameter resonance for $\lambda \simeq 10^{-3}$.

Figure 6 shows the reheating temperature for the case in which $\langle\varphi\rangle=0$. In this case, the SM gauge symmetries are unbroken in the epoch of reheating. Thermalization cannot occur before the inflaton decay has completed. Due to $\langle\varphi\rangle=$

\footnotetext{
6 Reference [29] provides an example how the gravitino problem in high-scale SUSY is evaded in the context of mini-split SUSY. In comparison with [29], the gravitino mass is far heavier in this paper, and kinematically blocking is the solution to the overproduction of gravitino.
} 


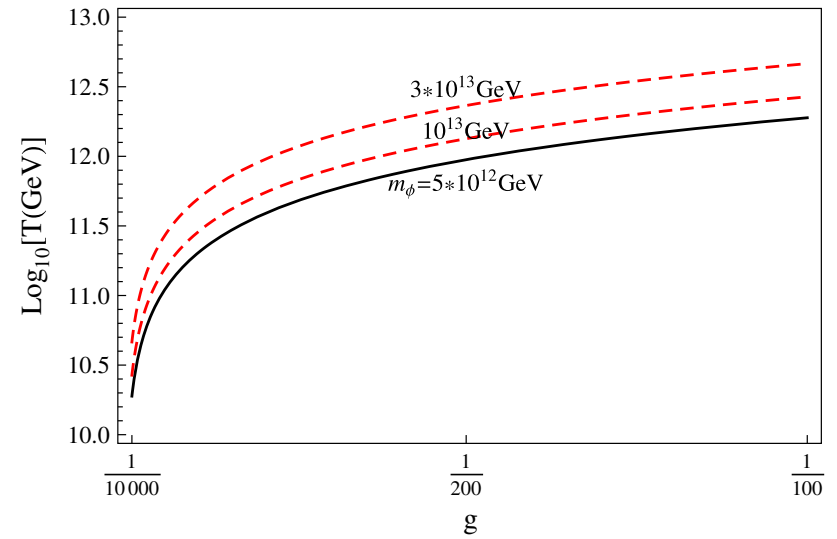

Fig. 6 Reheating temperature as a function of $g$ for $\langle\varphi\rangle=0$, with the definition $\Gamma_{d} \equiv g^{2} m_{\phi} / 8 \pi$

0 the $2 \rightarrow 3$ scatterings are already efficient when $H \simeq \Gamma_{d}$. The reheating temperature in this case is given by the standard expression: $T_{R} \simeq 0.3 \sqrt{\Gamma_{d} M_{P}}$. However, $\Gamma_{d}$ calculated via renormalizable couplings is rather different from Eq. (19) calculated via non-renormazible couplings. This difference between Figs. 4 and 6 is obvious. Typically, we have $T_{R} \geq$ $10^{10} \mathrm{GeV}$ in non-perturbative decay into MSSM and $T_{R} \leq$ $10^{10} \mathrm{GeV}$ in perturbative decay into SM.

Both Figs. 5 and 6 show that $T_{\mathrm{R}}$ is above $\sim 10^{9} \mathrm{GeV}$ but below $m_{3 / 2}$ in a wide range of parameter space. Due to the kinematically blocking effect this evades the overproduction of gravitino in conventional high-scale SUSY.

\section{Conclusions}

In the light of both LHC data and the Planck bound on $r$, high-scale SUSY is more favored compared with low-scale SUSY. In this paper, we discussed the implications of highscale SUSY on the early universe. In particular, we assumed that the inflation and visible sector have the same origin of SUSY breaking, and we derived model independent consequences based on this assumption. We find that the reheating temperature for the superpartner mass spectrum above $\mathcal{O}(100) \mathrm{TeV}$ might be below the value required by thermal leptogenesis if inflaton decays to its products perturbatively but above it if this decay is non-perturbatively instead. We also observed that the problem of gravitino overproduction can be evaded through kinematically blocking in a wide range of parameter space in the latter way.

As an illustration for the model building of inflation in the course of high-scale SUSY, in Sect. 3 we revise the simplest hybrid inflation, which includes a new linear term for the inflaton with a coefficient proportional to $m_{3 / 2}$. It is shown that this term significantly affects the choices on the initial condition of the inflaton fields. We found that with the assumption the simplest hybrid inflation is consistent with the present experimental data for $r$ of order $10^{-5}$.
Under our assumption only the dark matter is a light SUSY state with mass near the weak scale [30], which is the target of the LUX and Xenon experiments, etc. Hopefully, it can be addressed in the near future.

Acknowledgments We would like to thank J.-h. Huang for discussion. This work is supported in part by the Fundamental Research Funds for the Central Universities under Grant No. CQDXWL-2013-015.

Open Access This article is distributed under the terms of the Creative Commons Attribution 4.0 International License (http://creativecomm ons.org/licenses/by/4.0/), which permits unrestricted use, distribution, and reproduction in any medium, provided you give appropriate credit to the original author(s) and the source, provide a link to the Creative Commons license, and indicate if changes were made.

Funded by $\mathrm{SCOAP}^{3}$.

\section{References}

1. G. Aad et al. [ATLAS Collaboration], Observation of a new particle in the search for the Standard Model Higgs boson with the ATLAS detector at the LHC, Phys. Lett. B 716, 1 (2012). arXiv:1207.7214 [hep-ex]

2. S. Chatrchyan et al. [CMS Collaboration], Observation of a new boson at a mass of $125 \mathrm{GeV}$ with the CMS experiment at the LHC, Phys. Lett. B 716, 30 (2012). arXiv:1207.7235 [hep-ex]

3. S.P. Martin, A supersymmetry primer. Adv. Ser. Direct. High Energy Phys. 21, 1 (2010). arXiv:hep-ph/9709356

4. J.L. Feng, Naturalness and the status of supersymmetry, Ann. Rev. Nucl. Part. Sci. 63, 351 (2013). arXiv:1302.6587 [hep-ph]

5. P.A.R. Ade et al. [Planck Collaboration]. arXiv:1502.02114 [astroph.CO]

6. P.A.R. Ade et al. [Plank Collaboration], Planck 2013 results. XVI. In: Cosmological Parameters. arXiv:1303.5076 [astro-ph.CO]

7. P.A.R. Ade et al. [Plank Collaboration], Planck 2013 results. XXII. In: Constraints on Inflation. arXiv:1303.5082 [astro-ph.CO]

8. J.R. Espinosa, G.F. Giudice, E. Morgante, A. Riotto, L. Senatore, A. Strumia, N. Tetradis, The cosmological Higgstory of the vacuum instability. arXiv:1505.04825 [hep-ph]

9. S. Zheng, Can Higgs inflation be saved with high-scale supersymmetry? arXiv:1504.08093 [hep-ph]

10. Q. Shafi, J.R. Wickman, Phys. Lett. B 696, 438 (2011). arXiv:1009.5340 [hep-ph]

11. T. Higaki, K.S. Jeong, F. Takahashi, JHEP 1212, 111 (2012). arXiv: 1211.0994 [hep-ph]

12. D.H. Lyth, What would we learn by detecting a gravitational wave signal in the cosmic microwave background anisotropy? Phys. Rev. Lett. 78, 1861 (1997). hep-ph/9606387

13. K. Nakayama, F. Takahashi, T.T. Yanagida, Constraint on the gravitino mass in hybrid inflation. JCAP 1012, 010 (2010). arXiv: 1007.5152 [hep-ph]

14. G.R. Dvali, Q. Shafi, R.K. Schaefer, Large scale structure and supersymmetric inflation without fine tuning. Phys. Rev. Lett. 73, 1886 (1994). arXiv:hep-ph/9406319

15. A.D. Linde, Hybrid inflation. Phys. Rev. D 49, 748 (1994). arXiv:astro-ph/9307002

16. B.A. Bassett, S. Tsujikawa, D. Wands, Inflation dynamics and reheating. Rev. Mod. Phys. 78, 537 (2006). arXiv:astro-ph/0507632

17. A. Mazumdar, J. Rocher, Particle physics models of inflation and curvaton scenarios. Phys. Rept. 497, 85 (2011). arXiv: 1001.0993 [hep-ph] 
18. L. Kofman, A.D. Linde, A.A. Starobinsky, Phys. Rev. Lett. 73, 3195 (1994). arXiv:hep-th/9405187

19. L. Kofman, A.D. Linde, A.A. Starobinsky, Towards the theory of reheating after inflation. Phys. Rev. D 56, 3258 (1997). arXiv:hep-ph/9704452

20. J. Garcia-Bellido, A.D. Linde, Preheating in hybrid inflation. Phys. Rev. D 57, 6075 (1998). arXiv:hep-ph/9711360

21. R. Allahverdi, A. Mazumdar, Reheating in supersymmetric high scale inflation. Phys. Rev. D 76, 103526 (2007). arXiv:hep-ph/0603244

22. S. Davidson, S. Sarkar, Thermalization after inflation. JHEP 0011, 012 (2000). arXiv:hep-ph/0009078

23. R. Allahverdi, M. Drees, Thermalization after inflation and production of massive stable particles. Phys. Rev. D 66, 063513 (2002). arXiv:hep-ph/0205246

24. M. Dine, L. Randall, S.D. Thomas, Supersymmetry breaking in the early universe. Phys. Rev. Lett. 75, 398 (1995). arXiv:hep-ph/9503303
25. M. Dine, L. Randall, S.D. Thomas, Baryogenesis from flat directions of the supersymmetric standard model. Nucl. Phys. B 458, 291 (1996). arXiv:hep-ph/9507453

26. M.K. Gaillard, H. Murayama, K.A. Olive, Preserving flat directions during inflation. Phys. Lett. B 355, 71 (1995). arXiv:hep-ph/9504307

27. R. Allahverdi, A. Mazumdar, Supersymmetric thermalization and quasi-thermal universe: consequences for gravitinos and leptogenesis. JCAP 0610, 008 (2006). arXiv:hep-ph/0512227

28. M. Kawasaki, F. Takahashi, T.T. Yanagida, Gravitino overproduction in inflaton decay. Phys. Lett. B 638, 8 (2006). arXiv:hep-ph/0603265

29. J. Fan, B. Jain, O. Ozsoy, Heavy gravitino and split SUSY in the light of BICEP2. JHEP 07, 073 (2014). arXiv:1404.1914 [hep-ph]

30. S. Zheng, On dark matter selected high-scale supersymmetry. JHEP 1503, 062 (2015). arXiv:1409.2939 [hep-ph] 